\title{
Analysis the influence of pulse-to-pulse stability of modulator on high-power microwave output of pulsed klystron
}

\author{
Yongfang $\mathrm{Liu}^{1}\left(\mathbb{D} \cdot\right.$ Hiroshi Matsumoto ${ }^{2} \cdot \mathrm{Lin}^{\mathrm{Li}}{ }^{3} \cdot \mathrm{Ming} \mathrm{Gu}^{1}$
}

Received: 7 August 2021 / Accepted: 19 November 2021

Published online: 04 December 2021

(c) The Author(s) 2021 OPEN

\begin{abstract}
X-ray free electron laser (XFEL) facility based on electron linear accelerator (LINAC) is regarded as one kind of the fourthgeneration light source with the characteristics of high intensity, exceptional brightness, ultrashort pulse duration, and spatial coherence. In electron linear accelerator, energy of beam bunches is provided by high-power electromagnetic microwaves which are generated by a microwave tube called klystron. The stability of beam voltage of klystron occupies a key position in both the stability of output RF (Radio Frequency) power and the jitter of output RF phase, furthermore, it plays an extremely important role in beam energy stability of electron linear accelerator. In this paper, high power RF fluctuation and phase jitter of klystron output caused by beam voltage instability of klystron are analyzed and calculated. Influence of klystron beam voltage instability on beam energy gain in linear accelerator have also been further analyzed and calculated. The calculating procedure is particularly valuable for us to understand the relationship between pulse modulator stability and beam energy gain fluctuations. Finally, relevant experimental results measured by Shanghai Soft $\mathrm{X}$-ray Free Electron Laser Test Facility (SXFEL-TF) is presented.
\end{abstract}

Keywords High-power microwave $\cdot$ Pulse modulator $\cdot$ Klystron · LINAC · Stability · Beam energy fluctuation · Phase tolerance

\section{Introduction}

$\mathrm{X}$-ray free electron laser (XFEL) [1, 2] is considered to be the next generation light source, which involves potentially higher brilliance, a higher degree of coherence, and ultrashort pulses [3]. There are many XFEL facilities built or planned around the world, such as XFEL in Europe, LCLS-II in the USA, SACLA in Japan, FLASH in Germany, SwissFEL in Switzerland, MAX-IV in Sweden, PAL X-FEL in South Korea, etc.[4]. China's high-gain free electron laser (FEL) research started in late 1990s at Shanghai Institute of Applied Physics (SINAP). Shanghai Deep Ultraviolet-Free Electron Laser Facility (SDUV-FEL) is the first test bench for
HGHG FEL scheme [5]. In the past decade, Dalian Coherent Light Source (DCLS), an EUV-FEL user facility, based on a $300 \mathrm{MeV}$ linac were constructed [6]. Shanghai Soft $\mathrm{X}$-ray Free Electron Laser Test Facility is the first coherent $\mathrm{X}$-ray light source in China based on high-gain harmonic generation (HGHG) and echo-enabled harmonic generation (EEHG) scheme. SXFEL-TF use an $840 \mathrm{MeV}$ electron linac to generate $8.8 \mathrm{~nm}$ soft X-ray FEL radiation [7]. A high repetition-rate hard X-ray FEL facility based on a continuous wave superconducting linear accelerator will be plan to construct in the next ten years [8].

SXFEL-TF linac is based on normal conducting accelerating structure using traveling microwaves to accelerate

\footnotetext{
$\triangle$ Ming Gu, guming@zjlab.org.cn| ${ }^{1}$ Shanghai Advanced Research Institute, Chinese Academy of Sciences, Shanghai 201412, China. ${ }^{2} \mathrm{High}$ Energy Accelerator Research Organization, Tsukuba, Ibaraki 3050801, Japan. ${ }^{3}$ School of Internet of Things, Jiangxi Teachers College, Yingtan 335000, Jiangxi, China.
}

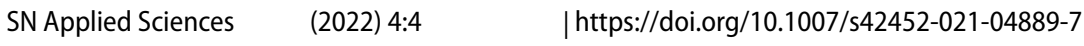


electrons [9]. The SXFEL-TF linac consists of an injector and a main linac. The injector consists of an S-band photocathode Radio Frequency (RF) gun, two S-band accelerating structure units, an X-band linearizer, and the main linac includes one S-band accelerating structure unit and six C-band accelerating structure units. The repetition rate is $10 \mathrm{~Hz}$. The most common layout of microwave acceleration structure unit is shown as Fig. 1. The klystron modulated by pulse modulator and pulse transformer converts electrical energy to microwaves. Microwaves feed into the accelerating structure by waveguide and Stanford Linac Energy Doubler(SLED). Beam energy comes from the microwaves output of klystron. The main linac use C-band accelerating structure accelerates beam energy up to $840 \mathrm{MeV}$ [10].

Figure 2 shows the picture of C-band klystron and pulse modulator. The microwave system of C-band accelerator structure used a $110 \mathrm{MW}$ pulse modulator and a $50 \mathrm{MW}$

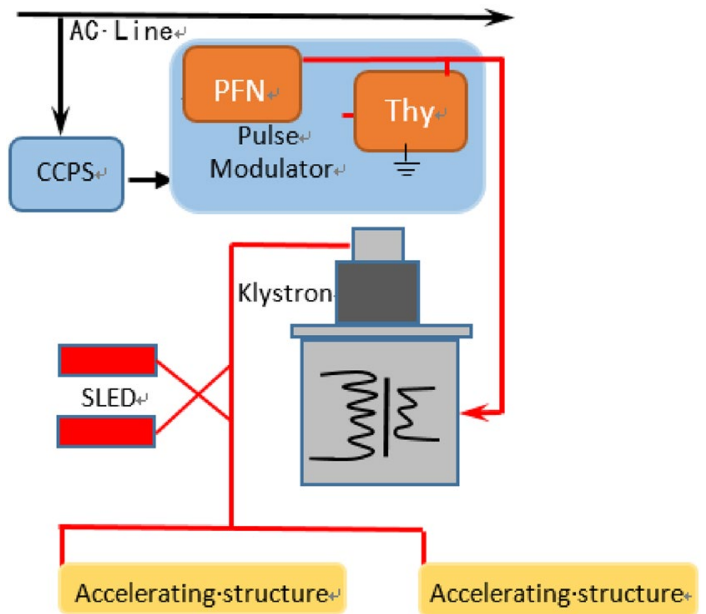

Fig. 1 The sketch of microwave acceleration unit for SXFEL klystron. $110 \mathrm{MW}$ pulse modulator based on Pulse Forming Network (PFN) and thyratron. Capacitor Charging Power Supply (CCPS) charges PFN to a set voltage value, and then thyratron switches on the discharge circuit, pulse waveform generates at the primary side of pulse transformer. Pulse transformer accomplishes increasing the voltage level, matching impedances, and polarity inversion. The stability of beam energy for electron linear accelerator depends mainly upon the beam voltage of klystron [11]. Beam voltage of klystron is provided by transformer's secondary coil of pulse modulator. Pulse modulator and klystron are two crucial equipment, which affects the highpower microwave output of klystron in two ways [12]. The first one is the stability of pulse modulator's high voltage, which will lead to fluctuation of microwave amplitude of klystron RF output [13]. The second one is the imperceptible change of pulse modulator's voltage, which will bring about tolerance of microwave phase of klystron RF output [14]. It is the pulse-to-pulse instability of modulator that will cause the high-power microwave amplitude fluctuation and phase tolerance of klystron, and thus cause the change of beam energy gain [15]. This paper describes each of these in detail by mathematics method. At the end we found a special phase point, where the beam energy gain is insensitive to the modulator voltage fluctuation.

\section{Influence of beam voltage stability on klystron output waveform}

\subsection{Principle of klystron}

Klystron is a microwave amplifier used for providing microwave power in higher energy (greater than $12 \mathrm{MeV}$ ) linear accelerators [16]. Klystron is one of the primary means of
Fig. 2 The picture of C-band klystron and pulse modulator

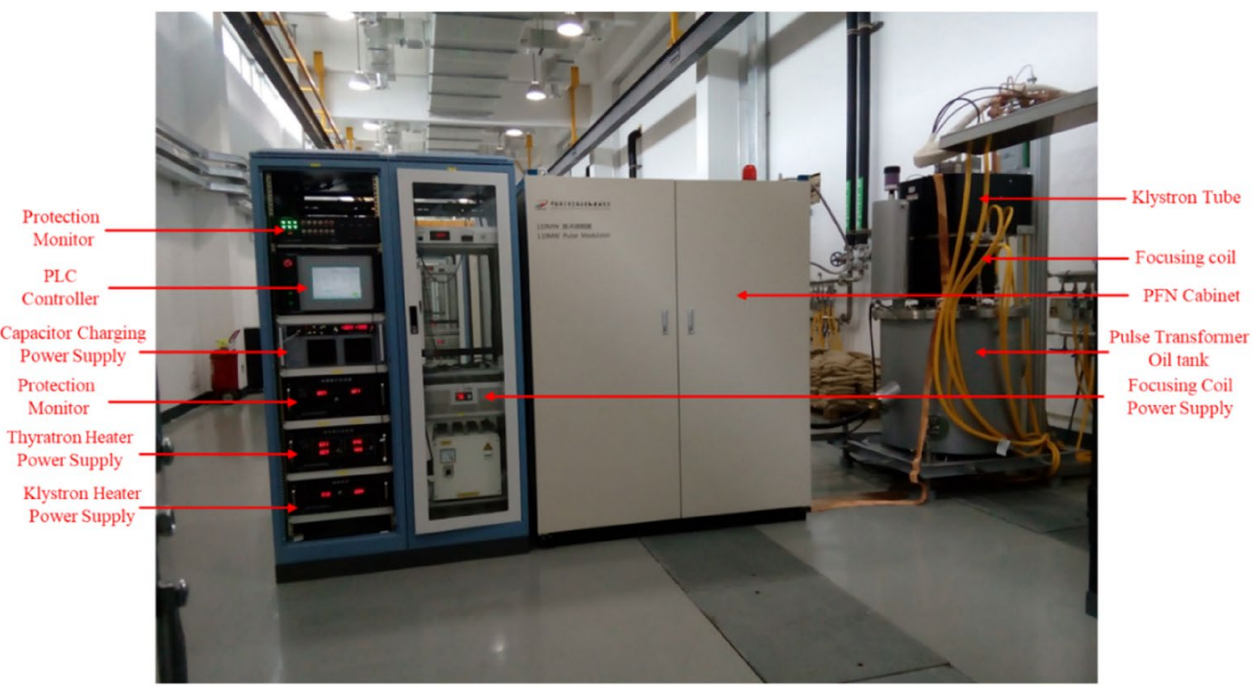


generating high power at UHF and above, which is used to generate high power microwave by means of velocity modulation and density modulation principle [17]. Klystron is a linear-beam vacuum device which is used as a narrow bandwidth amplifier for high radio frequencies. After the drift space, the density-modulated beam induces current in the output resonator [18]. Figure 3 shows the schematic diagram of an elementary two-cavity klystron tube.

The working principle of klystron can be described as follow.

The cathode(5) is heated by a heating filament(6) and then the electron beams injected from the cathode are first accelerated by high voltage electric field provided by pulse modulator power supply(7) before entering the buncher grids and arrive at the buncher with uniform velocity [19].

The low-level driving microwaves coupled into the buncher cavity resonator(8) generate an additional local electric field. The direction of the electric field changes with the frequency of the low-level driving microwaves coupled into the buncher. These changes alternately accelerate and decelerate the electrons of the beam passing through the grids. The electrons that pass through the buncher cavity resonator at zero points of the electric field pass through with unchanged velocity; those that pass through the positive half cycles of the electric field experience an increase in velocity; those passing through the negative half cycles of the electric field experience a decrease in velocity. The variation of the electron velocity in the drift length(3) is called velocity modulation [20]. With the velocity-modulated electron beams pass through the drift length, the faster electrons catch up with the slower electrons and form bunches. The velocity modulation turns to density modulation then [21].

At last, the bunching density-modulated beams enter a catcher cavity resonator(9) and induce a very high-power microwave in the output cavity structure. The high-power microwaves are coupled out by an output waveguide. The

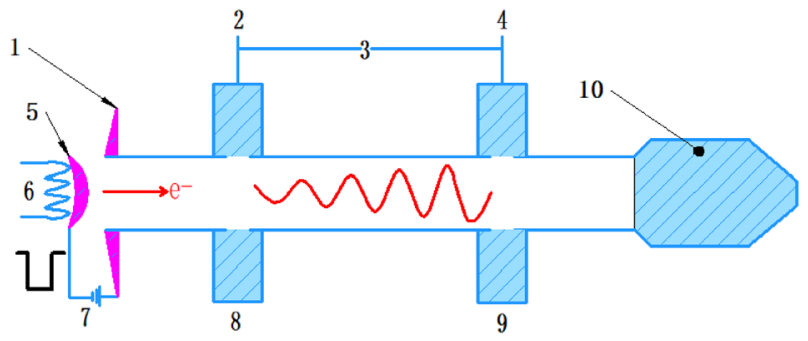

Fig. 3 A schematic diagram of an elementary two-cavity klystron tube; 1. Anode, 2. low level microwaves input, 3 . drift length, 4. high-power microwaves output, 5. Cathode, 6. heating filament, 7. pulse modulator power supply, 8 . buncher cavity resonator, 9 . catcher cavity resonator, 10 . electron beams collector electron beams are finally dumped in the electron beams collectors (10), where its energy converts into heat and $\mathrm{X}$-rays. The catcher cavity resonator is placed along the beam at a point where the bunching beams are fully formed. The location is determined by the transit time of the bunches at the natural resonant frequency of the cavities (the resonant frequency of the catcher cavity is the same as the buncher cavity) [22].

The multicavity klystron, which is similar to the principle of two-cavity klystron, reinforce the effect of velocity modulation and density modulation by additional intermediate cavities [23]. It makes even tighter electron bunching and high gain of klystron [24]. The multicavity klystron can increase the gain of the klystron or increase the bandwidth.

\subsection{Klystron output RF amplitude change caused by beam voltage fluctuation}

For a certain klystron, it holds the following characteristics:

\subsubsection{The perveance of klystron}

Perveance is a key parameter for klystron design. Due to space-charge-limited effect, the beam current is a specific function of the beam voltage which can be defined as the follow Eq. (1). The parameter $P$ termed as perveance depends on the geometry of the anode-cathode structure of the klystron gun. For a specific klystron, $P$ P P is a constant parameter,

$P=\frac{I_{\mathrm{K}}}{V_{\mathrm{K}}^{1.5}}=\frac{I_{\mathrm{K}}}{V_{\mathrm{K}}} V_{\mathrm{K}}^{-0.5}=\frac{V_{\mathrm{K}}^{-0.5}}{R_{\mathrm{k}}}$

where $I_{\mathrm{K}}$ is the beam current of klystron in amps, $V_{\mathrm{K}}$ is the beam voltage of klystron in volts, $R_{\mathrm{k}}$ is the equivalent impedance of klystron in ohm. $R_{\mathrm{k}}$ is defined as the ratio of the beam voltage $V_{\mathrm{K}}$ to beam current $I_{\mathrm{K}}$.

\subsubsection{The efficiency of klystron}

$\eta$ is defined as the ratio of the microwave output power $P_{0}$ to the electron beam power which equals to the product of beam current. It can be can be expressed as Eq. (2).

$\eta=\frac{P_{\mathrm{o}}}{P_{\mathrm{I}}}=\frac{\frac{1}{2 \pi} \int_{0}^{2 \pi} V_{\text {out }} \cos (\omega t) I_{\text {out }} d(\omega t)}{V_{\mathrm{K}} I_{\mathrm{K}}}$

where $\omega t$ is the phase of high-power RF output, $\omega t=\omega\left(t_{0}+T\right)=\phi_{0}+\tau(\phi), \tau(\varphi)$ is electron transit time from input to output, $P_{0}$ is the RF output power of klystron, $P_{l}$ is the electron beam power, $V_{\text {out }}$ is the RF output voltage amplitude, $I_{\text {out }}$ is the RF output current amplitude. 


\subsubsection{The output power of klystron}

The calculation of klystron output power of can be expressed as Eq. (3) [25]. The klystron output RF amplitude of electric field can be calculated according to Ohm's Law[26]. It can be expressed as Eq. (4).

$P_{\mathrm{RF}}=\eta I_{\mathrm{K}} V_{\mathrm{K}}=\eta P V_{\mathrm{K}}^{2.5}$

$V_{\mathrm{RF}}=\sqrt{P_{\mathrm{RF}} R_{\mathrm{L}}}=\sqrt{\eta P R_{\mathrm{L}} V_{\mathrm{K}}^{2.5}}$

where $P_{R F}$ is the microwave output power of klystron in watt, $\eta$ is the efficiency of klystron, $R_{L}$ is the load impedance of klystron in ohm, $P$ is the perveance of klystron.

\subsubsection{RF amplitude sensitivity to beam voltage fluctuation}

From Eq. (4) we can obtain that output RF amplitude of klystron is a specific function of beam voltage. As discussed above, $\eta$ is also a specific function of beam voltage. Figure 4 shows the test result of the relationship between efficiency and beam voltage for a specific klystron (E37115). As indicated in Fig. 4, the efficiency sensitivity of a klystron to beam voltage variation in low level voltage linear region is larger than in saturation region. $\frac{d \eta}{d v_{k}}$ is very small, almost zero, in saturation state. Therefore, when we calculate RF amplitude sensitivity to beam voltage fluctuation, we treat $\eta$ as a constant. We can substitute Eqs. (3) and (4) into their differential equation, respectively. Consequently, the sensitivity of output RF power and amplitude of a klystron can be calculated by Eqs. (5) and (6). That is to say, RF power stability is two and a half times in comparison with the beam voltage stability. And RF amplitude is one point two five times in comparison with the beam voltage stability. Figure 5 shows the above

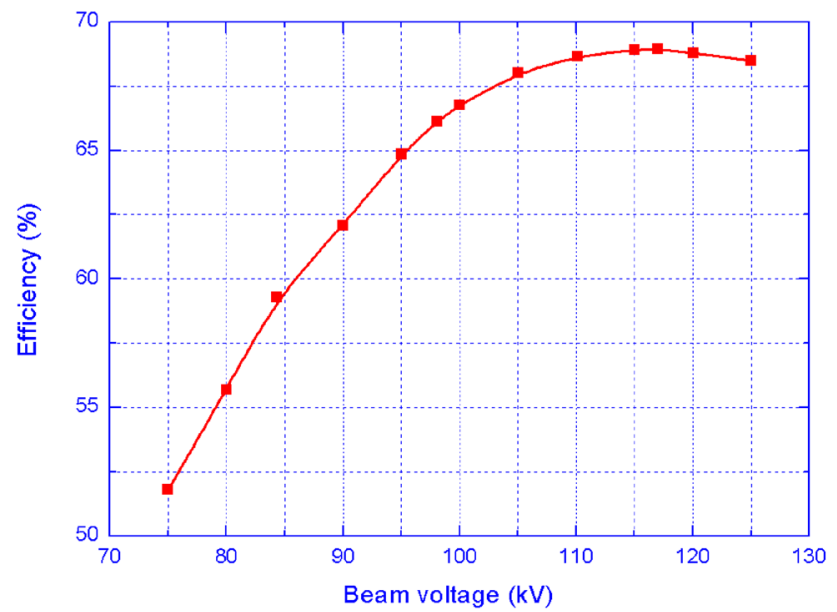

Fig. 4 Test data of efficiency and beam voltage of a klystron relationship between RF output stability and beam voltage stability. It is important to point out that there will be a correction parameter if we do not treat efficiency as a constant but as a specific function of beam voltage.

$\frac{d P_{\mathrm{RF}}}{P_{\mathrm{RF}}}=2.5 \frac{\eta P V_{\mathrm{K}}^{1.5}}{\eta k V_{\mathrm{K}}^{2.5}} d V_{\mathrm{k}}=2.5 \frac{d V_{\mathrm{k}}}{V_{\mathrm{k}}}$

$\frac{d V_{\mathrm{RF}}}{V_{\mathrm{RF}}}=1.25 \frac{\sqrt{\eta P R_{\mathrm{L}}}}{\sqrt{\eta P R_{\mathrm{L}} V_{\mathrm{K}}^{2.5}}} V_{\mathrm{K}}^{0.25} d V_{\mathrm{k}}=1.25 \frac{d V_{\mathrm{k}}}{V_{\mathrm{k}}}$

It should be noted that, Eqs. (5) and (6) are based on $\eta$ as a constant, which means klystron operates in the saturated region of beam voltage. This kind of situation is the most common work situation for klystron application. When the klystron operates in the unsaturated region of beam voltage, the efficiency $\eta$ is a specific function of beam voltage. Then Eq. (6) turns to Eq. (7). It can be seen that, for the influence of beam voltage on RF amplitude sensitivity, there will add an impact factor of $\frac{k V_{K}}{2 \eta}$ when the klystron operates in the saturated region than the unsaturated region of beam voltage.

$\frac{d V_{\mathrm{RF}}}{V_{\mathrm{RF}}}=\frac{V_{\mathrm{K}} d \eta}{2 \eta V_{\mathrm{K}}}+1.25 \times \frac{d V_{\mathrm{K}}}{V_{\mathrm{K}}}=\frac{k V_{\mathrm{K}}}{2 \eta} \times \frac{d V_{\mathrm{K}}}{V_{\mathrm{K}}}+1.25 \times \frac{d V_{\mathrm{K}}}{V_{\mathrm{K}}}$

where $k$ is the slope of curve in Fig. 4.

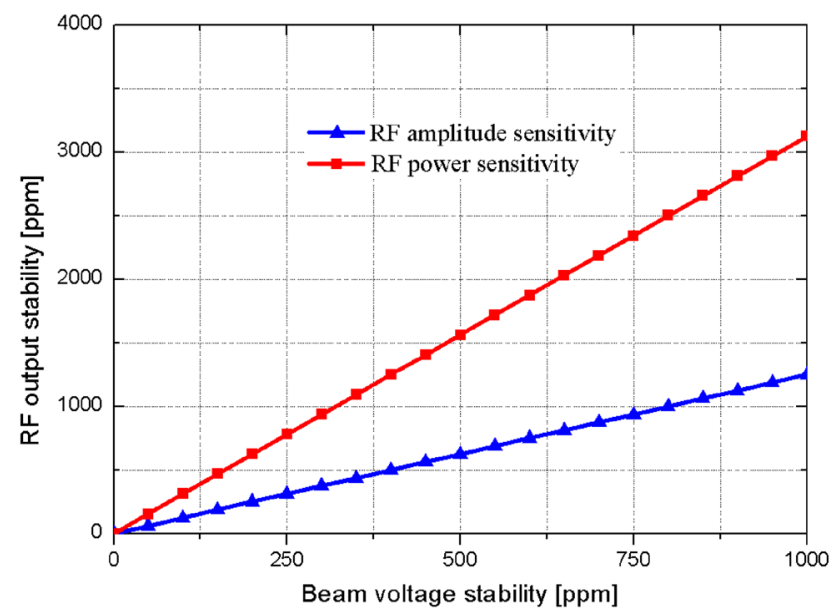

Fig. 5 Relationship between RF output stability and beam voltage stability 


\subsection{Klystron output RF phase variation cause by beam voltage fluctuation}

According to the klystron principle [27], Electron beams generated by heated cathode get kinetic energy by highpower voltage of pulse modulator and then modulated by a low-level RF signal coupled into input cavity. If the pulse voltage of modulator makes a slight variation, the velocity of the electron beam will also change, which will lead to the difference of the arriving time at the input cavity. After being velocity-modulated by the input RF signal, the time consumed for going across the drift space will fluctuate, and finally the time to reach the output cavity will also change. Thus, RF signal output phase fluctuations. For the convenience of derivation, we take the two-cavity klystron tube shown in Fig. 3 as an example. The phase variation caused by beam voltage fluctuation can be calculated by following procedures.

The energy of electron beams accelerated by the cathode pulse voltage is given by the following Eq. (8).

$e V=(\gamma-1) m c^{2}$

So $\gamma=\frac{\mathrm{eV}}{\mathrm{mc^{2 }}}+1$

where e is the charge of the electron, e is $1.6 \times 10-19 C, V$ is cathode pulse voltage in volts, eV is the energy of electron beams in electron volt, $\mathrm{m}$ is the mass of the electron, $c$ is the velocity of light, $\gamma$ is Lorentz factor which can be calculated by Eq. (9).

The time consumed for passing through drift length can be calculated by Eq. (10).

$\mathrm{t}=\frac{L}{C \beta}=\frac{L}{C \sqrt{1-\frac{1}{\gamma^{2}}}}$

where $\beta=\sqrt{1-\frac{1}{\gamma^{2}}}, \mathrm{~L}$ is the length of drift space, $\mathrm{C}$ is the velocity of light.

The phase delay of RF signal induced by the electron beam in the output cavity through the drift segment is calculated by Eq. (11).

$\theta=w t=\frac{w L}{C \sqrt{1-\frac{1}{\gamma^{2}}}}$

where $\omega$ is the angular frequency of the RF signal, $L$ is the length of drift space.

We use $\sin (w t)$ to express the input RF signal, then the output RF signal can be given by Eq. (12).

$A \sin (w t+\theta)$ where $A$ is the amplitude gain, $\theta$ is the phase delay after passing through drift length. As discussed, there will not be only amplitude gain change but also phase delay change when pulse voltage has a fluctuation. Thus, the output RF signal can be expressed as Eq. (13).

$(A+\Delta A) \sin (\mathrm{wt}+\theta+\Delta \theta)$

The counting process about $(\mathrm{A}+\Delta \mathrm{A})$ has been discussed in part 2.2 in this paper. Klystron output RF phase variation caused by beam voltage fluctuation can be deduced by a differential method. Equation (14) shows the differential result of phase delay.

$d \theta=-\frac{e \omega \mathrm{LV}}{m c^{3}\left(\gamma^{2}-1\right)^{3 / 2}} \times \frac{\mathrm{dV}}{V}$

where $d \theta$ is phase variation, $\frac{d V}{V}$ is the stability of beam voltage of klystron. Equation (14) is on behalf of the relationship between klystron output RF phase variation and beam voltage fluctuation.

In non-relativistic case, the energy of electron beams accelerated by the cathode pulse voltage adopts the following Eq. (15) based on classical electrodynamics. Thus, Eqs. (8) and (9) should be corrected to Eqs. (15) and (16). RF phase variation cause by beam voltage fluctuation can be deduced by calculating the derivative of Eq. (17). Equation (18) shows the phase variation expression in non-relativistic.

$\mathrm{eV}=\frac{1}{2} \mathrm{mv} \mathrm{v}^{2}$

$t=\frac{L}{V}=\frac{L}{\sqrt{\frac{2 e V}{m}}}$

$\theta=w t=w \frac{L}{\sqrt{\frac{2 e V}{m}}}$

$d \theta=-\frac{1}{2} \frac{\mathrm{wL}}{\sqrt{\frac{2 e V}{m}}} \frac{\mathrm{dV}}{V}=-\frac{1}{2} \theta \frac{\mathrm{dV}}{V}$

For megawatt pulsed klystron, beam voltage usually operates in hundreds of kilovolts [28]. It is more reasonable to use Eq. (14). Figure 6 shows the phase tolerance caused by beam voltage fluctuation when beam voltage works at $200 \mathrm{kV}, 250 \mathrm{kV}, 300 \mathrm{kv}, 350 \mathrm{kV}$, and $400 \mathrm{kV}$. It should be pointed out that Fig. 6 is calculated by Eq. (14). Equation (14) is the differentiation of phase delay function shown as Eq. (11). It is only suited to the estimation of very small RF phase jitter. 


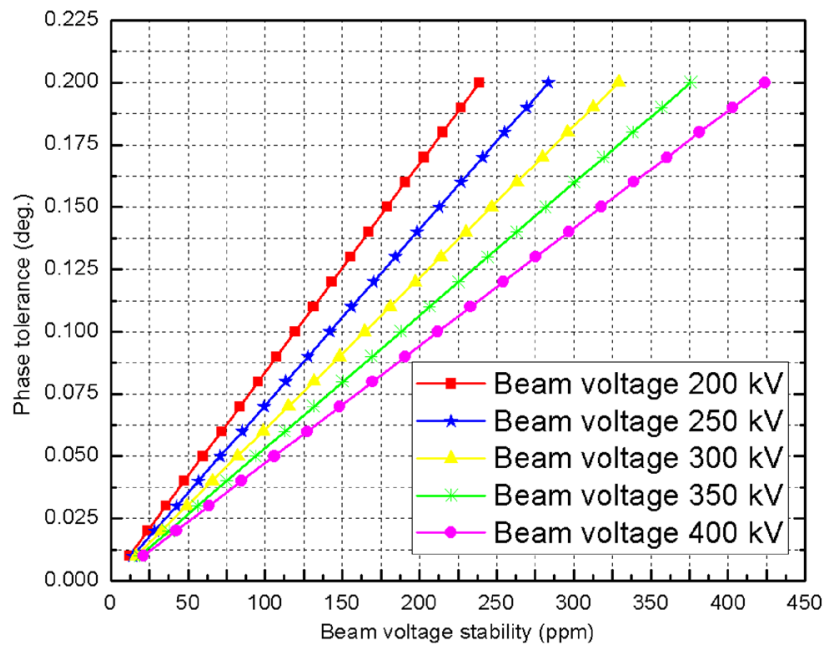

Fig. 6 Phase tolerance vs. beam voltage stability of a C-band klystron

\section{Influence of klystron beam voltage stability on beam energy gain fluctuation}

According to the working principle of accelerating structure, electron linear accelerator has two accelerating modes: travelling wave accelerator and standing wave accelerator. The travelling wave electron linear accelerator can be divided into constant impedance accelerating structure and constant gradient accelerating structure. SXFEL applies a traveling wave electron linear accelerator with equal gradient acceleration structure [29]. This section mainly discusses the effect of high voltage jitter of pulse modulator on the beam energy of accelerator.

The energy gained by electron on per unit accelerating structure length can be expressed as Eq. (19).

$E_{\mathrm{z}}(\mathrm{v} / \mathrm{m})=\mathrm{e} \sqrt{2 \alpha R_{\mathrm{m}} P_{\mathrm{RF}}} \sin \theta$

where $\alpha$ is attenuation coefficient, $R_{m}$ is the waveguide characteristic impedance. $P_{R F}$ and $\theta$ are both function of beam voltage, which are shown as Eq. (4) and Eq. (11). Equation (20) shows the differential result of Eq. (19).

$\frac{\Delta E_{\mathrm{z}}(\mathrm{V} / \mathrm{m})}{E_{\mathrm{z}}(\mathrm{V} / \mathrm{m})}=\left(1.25-\frac{e w L V}{\mathrm{mc}^{3} \beta^{3} \gamma^{3}} \cot \theta\right) \frac{\Delta V}{V}$

where Eq. (20) indicates the relationship between the energy variation of electron beam and the high voltage stability of pulse modulator.

After forming mathematical calculation on the energy variation of electron beam, we found a special phase point, where the beam energy gain is insensitive to the modulator voltage fluctuation. This phase can counteract the fluctuations of both RF amplitude and RF phase. It can be figure out by setting Eq. (20) to zero. It depends on some

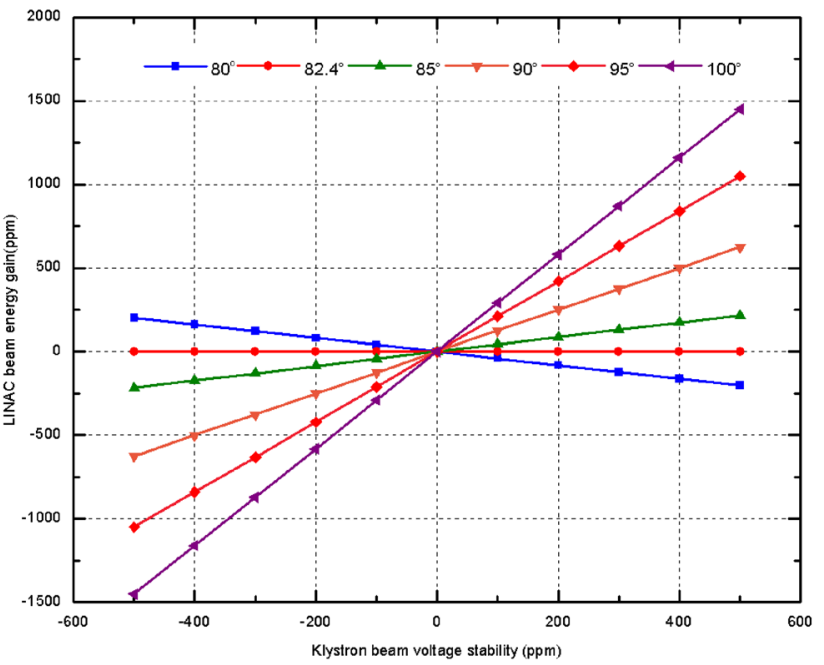

Fig. 7 The relationship between the electron beam energy and the stability of the modulator pulse under different phases

parameters of klystron such as rated beam voltage, drift length of klystron efficiency. For C-band klystron, we can calculate this phase is 82.4 degree before the crest of sinusoidal signal. Table 1 shows the calculated result of different phase point. Figure 7 shows the relationship between the electron beam energy and the stability of the modulator pulse under different phases.

\section{Stability result of pulse modulator and klystron output for SXFEL}

Figure 8 shows the high-power microwaves output test schematic diagram of klystron. Directional coupler is widely used in microwave system to measure the output power of the klystron. Microwave detectors are used to convert amplitude-modulated microwave signals to baseband signals. HD4104 is high performance oscilloscope with up to $10 \mathrm{GHz}$ sample rate, 12-bit ADCs, high signal-to-noise input amplifiers and a low-noise system architecture. We use HDO4104 to obtain the oscillogram and transformer oscillogram data to LabVIEW to further analysis. Figure 9 shows the test results of RF amplitude stability for pulse klystron. The RF amplitude stability for pulse klystron is $0.31 \%$.

Figure 10 shows pulse-to-pulse stability test system of pulse modulator. Trigger generator generate timing signal to pulse modulator and oscilloscope. A $20 \mathrm{MHz}$ in-house made common mode noise filter and a differential amplifier makes front signal processing to the pulse voltage. Figure 11 shows the test results of pulse-to-pulse stability for pulse modulator. The pulse-to-pulse stability for pulse modulator is $0.19 \%$. 
Fig. 8 The high-power microwaves output test schematic diagram of klystron

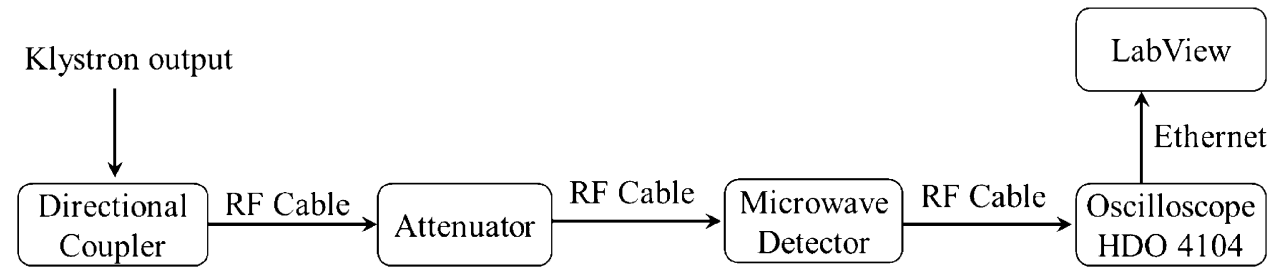

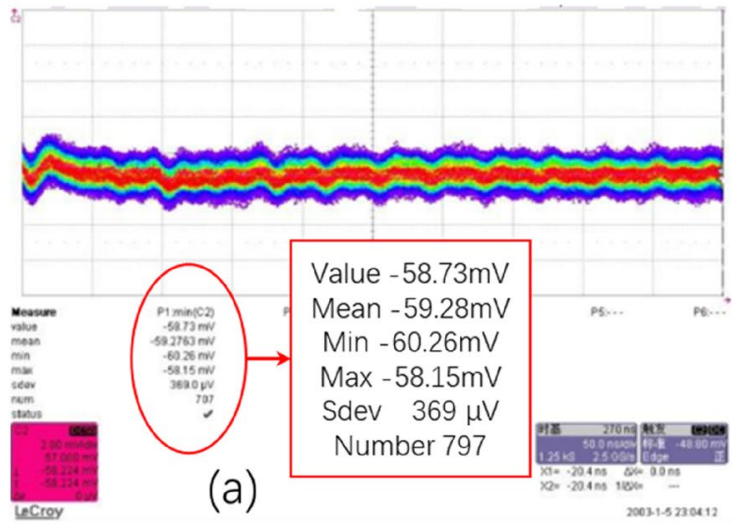

Fig. 9 Test results of RF Amplitude stability for pulse klystron. Running amplitude standard deviation of 797 consecutive pulses measured with oscilloscope (a) and Running amplitude standard

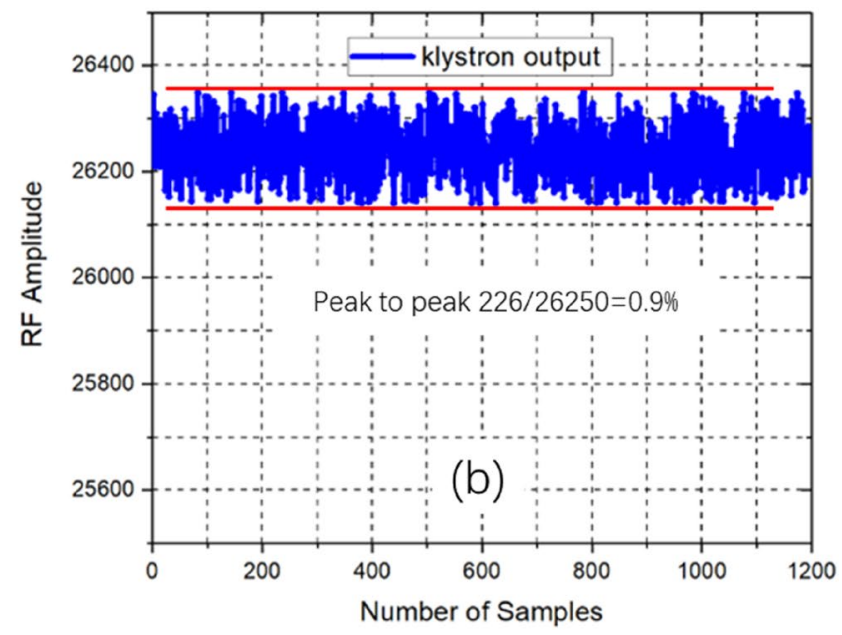

deviation of 1200 consecutive pulses measured with data acquisition system based on ADC
Fig. 10 Pulse-to-pulse stability test system of pulse modulator
Pulse Voltage

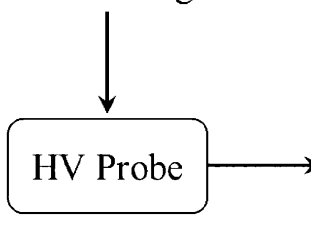

Common mode noise filter
$\mathrm{PC}$ LabView DG-645A Ethernet

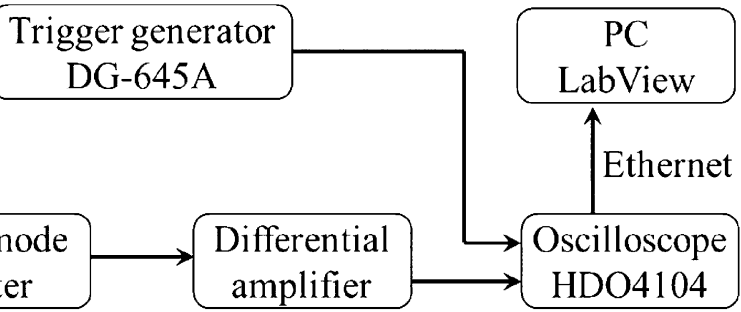

\section{Summary and discussion}

This paper analyzes the influence of klystron beam voltage stability on RF output in LINAC RF system. Beam voltage instability can cause amplitude and phase fluctuation of klystron RF output. It is 1.25 times for RF amplitude fluctuation compareds with beam voltage stability. RF phase variation caused by beam voltage fluctuation also can be calculated. For the constant gradient traveling wave accelerating structure, the relationship between klystron beam voltage stability and beam energy gain fluctuation in linear accelerator can be further derived.

The comprehensive effects of beam energy gain fluctuation were studied. In order to obtain the table beam energy, different phase point, slit arrangements with various slit directions, distribution and width are analyzed and discussed. Through the above analysis and comparisons, we can draw the following conclusions:

(1) The effect of RF phase jitter on beam energy can be negligible, when the beam operates at $90^{\circ}$ phase. At this point, the relationship between beam energy and pulse high voltage stability of the pulse modulator is 1.25 times.

(2) As the pulse high voltage increases, the RF output power of klystron increases, and vice versa, when the beam operates at $0-90^{\circ}$, while the RF phase decreases and sin decreases. In terms of electron beam energy, $\mathrm{RF}$ amplitude and phase changes caused by the high voltage of the modulator pulse cancel each other. 


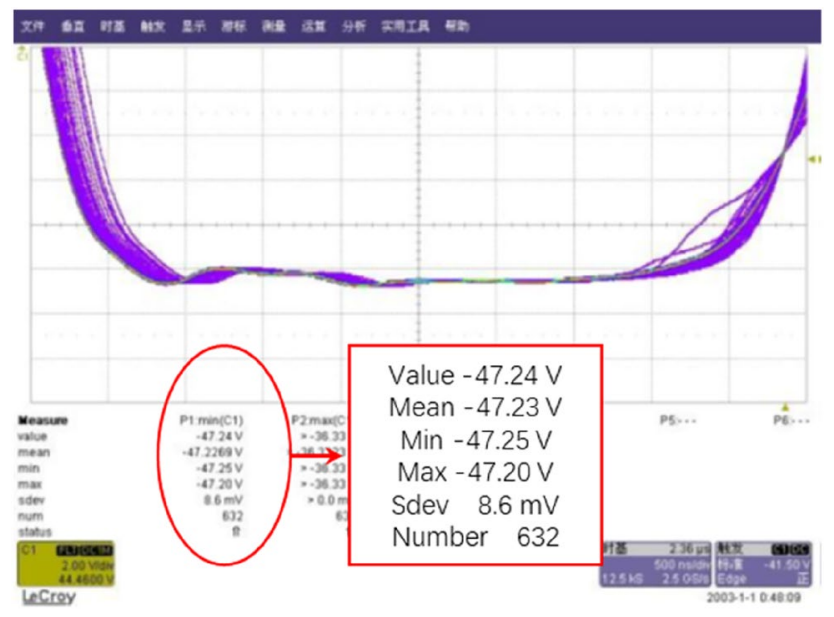

Fig. 11 Test results of pulse-to-pulse stability for pulse modulator. Running standard deviation of the amplitude of 632 consequent pulses measured simultaneously by oscilloscope

Table 1 calculated result of different phase point

\begin{tabular}{ll}
\hline Phase point & Calculated result \\
\hline $80^{\circ}$ & $\frac{\Delta E_{z}(v / m)}{E_{z}(v / m)}=-0.4 \frac{\Delta V}{V}$ \\
$82.4^{\circ}$ & $\frac{\Delta E_{z}(v / m)}{E_{z}(v / m)}=0$ \\
$85^{\circ}$ & $\frac{\Delta E_{z}(v / m)}{E_{z}(v / m)}=0.43 \frac{\Delta V}{V}$ \\
$90^{\circ}$ & $\frac{\Delta E_{z}(v / m)}{E_{z}(v / m)}=0.43 \frac{\Delta V}{V}$ \\
$95^{\circ}$ & $\frac{\Delta E_{z}(v / m)}{E_{z}(v / m)}=2.1 \frac{\Delta V}{V}$ \\
$100^{\circ}$ & $\frac{\Delta E_{z}(v / m)}{E_{z}(v / m)}=2.9 \frac{\Delta V}{V}$ \\
$80^{\circ}$ & $\frac{\Delta(v / m)}{E_{z}(v / m)}=-0.4 \frac{\Delta V}{V}$ \\
$82.4^{\circ}$ & $\frac{\Delta E_{z}(v / m)}{E_{z}(v / m)}=0$ \\
$85^{\circ}$ & $\frac{\Delta E_{z}(v / m)}{E_{z}(v / m)}=0.43 \frac{\Delta V}{V}$ \\
$90^{\circ}$ & $\frac{\Delta E_{z}(v / m)}{E_{z}(v / m)}=1.25 \frac{\Delta V}{V}$ \\
\hline
\end{tabular}

conclusions offer the theory sustain for pulse modulator designers. In addition, the research results have directive significance for accelerator operators to obtain LINAC beam stability with better properties.

Acknowledgements The authors would like to thank the other members of Pulse Technique group and Radio Frequency group in Shanghai Advanced Research Institute. The authors would like to thank the reviewers and editors for their hard work on this research.

Author contributions Yongfang. Liu conducted the review and wrote the manuscript. Ming. Gu check the manuscript grammar and help in enhancing the quality of the manuscript. Lin. Li made the experiments. Hiroshi. Matsumoto guided and contributed in discussions and supervision. All authors have read and agreed to the published version of the manuscript.

Funding Project supported by Shanghai Municipal Science and Technology Major Project (Grant No.2017SHZDZX02), the National Natural Science Foundation of China (No.12005282) and Youth Innovation Promotion Association of Chinese Academy of Sciences. Young Scientists Fund (No. 2021283) .

\section{Declarations}

Conflict of interest The authors declare no conflict of interest.

Open Access This article is licensed under a Creative Commons Attribution 4.0 International License, which permits use, sharing, adaptation, distribution and reproduction in any medium or format, as long as you give appropriate credit to the original author(s) and the source, provide a link to the Creative Commons licence, and indicate if changes were made. The images or other third party material in this article are included in the article's Creative Commons licence, unless indicated otherwise in a credit line to the material. If material is not included in the article's Creative Commons licence and your intended use is not permitted by statutory regulation or exceeds the permitted use, you will need to obtain permission directly from the copyright holder. To view a copy of this licence, visit http://creativecommons. org/licenses/by/4.0/.
(3) When the accelerator operates at $90-180^{\circ}$.The pulse high voltage increases the RF output power of klystron, while the RF phase decreases and the sine theta increases and vice versa. In terms of electron beam energy, RF amplitude and phase changes caused by high voltage of modulator pulse have superimposed effects on beam energy.

The experimental results basically tally with theoretical analysis and calculation results expatiated in this paper. Despite the limitations these are valuable in light of these conclusions analyzed in this paper. Generally, it is not easy for engineers to specify the evolution of the stability requirements from pulse modulator to beam energy in LINAC RF system. The present paper introduces the theory for the design of pulse modulator parameters in order to improve the LINAC performance. The

\section{References}

1. Kondratenko AM, Saldin EL (1980) Generation of coherent radiation by a relativistic electron beam in an ondulator. Synth Commun 51(3):1633-1642

2. Bonifacio R, Pellegrini C, Narducci LM (1984) Collective instabilities and high-gain regime in a free electron laser. Opt Commun 50(6):373-378

3. Feng C, Deng H-X (2018) Review of fully coherent free-electron lasers. Nucl Sci Tech 29(11):160. https://doi.org/10.1007/ s41365-018-0490-1

4. Pellegrini C (2017) X-ray free-electron lasers: from dreams to reality. Phys Scr 169:014004

5. Feng $C$, Zhang $M$, Chen JH, Deng HX, Gu Q, Wang D, Zhao ZT (2011) Design studies for cascaded HGHG and EEHG experiments based on SDUV-FEL. Proceedings of the 33rd International Free Electron Laser Conference, Shanghai, China

\section{SN Applied Sciences}


6. Haixiao DENG, Meng Z, Duan G et al (2014) Simulation studies on laser pulse stability for Dalian coherent light source. Chinese Phys. C 02:91-96

7. Zhao ZT, Feng C, Zhang KQ (2017) Two-stage EESHG for coherent hard X-ray generation based on a superconducting linac. Nucl Sci Tech 28:117. https://doi.org/10.1007/ s41365-017-0258-Z

8. Zhao ZT, Feng C, Zhang KQ (2017) Two-stage EEHG for coherent hard $\mathrm{X}$-ray generation based on a superconducting linac. Nucl Sci Tech 28(008):54-60

9. Meng Z, Qiang G (2011) New linear accelerator (Linac) design based on C-band accelerating structures for SXFEL facility. Chinese Phys. C 35(11):1066-1069

10. Liu YF, Matsumoto H, Gu M et al (2019) Analysis and optimization of high-power pulse transformer for SXFEL. Nucl Sci Tech 30:109. https://doi.org/10.1007/s41365-019-0626-y

11. Takayama K (1989) Stability of a klystron-type free-electron laser in the steady-state regime: Macroparticle approach. Phys Rev Lett 63(5):516

12. Park GS, Granatstein VL (1991) Phase stability of gyroklystron amplifier. IEEE Trans Plasma Sci 19(4):632-640

13. Sakaki $H$, Yoshikawa $H$, Hori T et al. (2002) Beam energy gain fluctuation in a linac caused by RF system noise. Nuclear Instruments \& Methods in Physics Research, Section A, (Accelerators, Spectrometers, Detectors and Associated Equipment), 480(2-3):403-411

14. Zeng R (2012) Investigation of feedback control for klystron ripple. Proceedings of the 3th International Particle Accelerator Conference, New Orleans, Louisiana, USA

15. Oh JS, Inagaki T, Shirasawa K et al. (2006) Stable RF phase insensitive to the modulator voltage fluctuation of the C-band Main Linac for SCSS XFEL. Proceedings of the 28th International Free Electron Laser Conference, BESSY, Berlin, Germany

16. Matveev NV (2010) High power pulse modulator for vacuum microwave devices. Proceedings of the International Conference on Actual Problems of Electron Devices Engineering. IEEE

17. Ahmad H, Yusoff N, Monajemi H et al (2019) Nickel phosphate as a C-band optical pulse modulator. Appl Phys B 125(7):132

18. Oh JS, Park SS, Han YJ et al. (2006) Design considerations for the stability improvement of klystron-modulator for PAL XFEL. Proceedings of the Particle Accelerator Conference. IEEE
19. Oh JS, Inagaki T, Shirasawa K et al. (2006) Sensitivity analysis of the beam energy of a C-band main linac for SCSS XFEL. Proceedings of the 3rd Annual Meeting of Particle Accelerator Society of Japan And the 31th Linear Accelerator Meeting in Japan, Sendai Japan, August pp 2-4

20. Wu Y, Li ZH, Xie HQ et al (2014) An S-band high gain relativistic klystron amplifier with high phase stability. Phys Plasmas 21(11):113107

21. Liu Y, Tong J, Gu M et al (2019) Design a precise stability controller for high power pulse modulator based on FPGA. J Phys Conf Ser 1350:012158

22. Kim DS, Lee BK, Park SS et al (2020) High-capacity capacitor charging power supply for a pulse modulator. J- Korean Phys Soc 76(7):547-550

23. Zhang, L, Zhu Z, Liu B et al (2015) Improving stability of pulse modulator technology. $<$ div class="NodiCopyInline" $>$ Annual Report of China Institute of Atomic Energy, 000(001), P.238-.

24. Hobson GS (2001) A high-stability pulse modulator for Gunn oscillators and amplifiers. J Phys E: Sci Instrum 10(4):359-360

25. Hwang J, Park S-J, Namkung W, Cho M (2016) Stability analysis of c-band 500-kw klystron with multi-cell output cavity. J Korean Phys Soc 69(6):940-947

26. Biswas BN, Chatterjee S, Choudhury B, Mallick SG (2015) Kinematic Principle of Reflex Klystron: A Necessary Revisit. Internatlonal Journal of electronlcs \& communlcatlon technology(IJECT) 6(3)

27. Fukuda S, Hayashi K, Maeda S et al (1999) Performance of a highpower klystron using a BI cathode in the KEK electron linac. Appl Surf Sci 146(1-4):84-88

28. Grudiev A, Yamamoto A (2013) Design and principles of linear accelerators and colliders: accelerating structures design and efficiency. Landolt-Börnstein, 21c

29. Savkin VY, Yakovlev DV (2015) A high-voltage pulse modulator for high-power microwave electronics. Instrum Exp Tech 58(6):741-744

Publisher's Note Springer Nature remains neutral with regard to jurisdictional claims in published maps and institutional affiliations. 